[Article]

\title{
支状嵌段聚醚的界面聚集行为及对原油乳状液的破乳作用
}

\author{
翟雪如 ${ }^{1}$ 刘 腾 $^{1}$ 徐桂英 ${ }^{1, *}$ 檀国荣 ${ }^{2}$ 吕 金金 $^{2}$ 张 健 $^{2}$ \\ ('山东大学胶体与界面化学教育部重点实验室, 济南 250100; 2中海油研究总院技术研发中心, \\ 海洋石油高效开发国家重点实验室, 北京 100027)
}

\begin{abstract}
摘要：合成了三种不同聚氧丙烯/聚氧乙烯(PPO/PEO)比例的含苯环支状嵌段聚醚, 通过界面张力、界面流 变、表面压以及对原油乳状液的破乳脱水效果的测定, 考察了其界面聚集行为和破乳作用对 PEO 含量和分子 量的依赖性, 并且对比研究了三种支状聚醚分子交联前后的破乳性能. 结果表明, PEO 含量高且分子量大者, 其单分子界面占据面积大, 在油/水界面达到吸附平衡的时间短, 其油/水界面扩张模量及扩张弹性均高于 PEO 含量较少者. 但是对原油乳状液的破乳脱水效果则是 PEO 含量居中的聚醚最好. 温度影响和交联与否的研究 表明, 交联并不能提高分子量较大的聚醚对原油乳状液的破乳效果, 温度对聚梄分子交联前后的破乳效果有不 同的影响规律. 本研究可为原油集输过程中化学品的选择与应用提供一定的依据.
\end{abstract}

关键词：支状嵌段聚梄；界面活性；界面流变性；破乳作用

中图分类号: 0648

\section{Aggregation Behavior of Branched Block Polyethers at Interface and Their Demulsification for Crude Oil Emulsion}

\author{
ZHAI Xue-Ru1 $\quad$ LIU Teng ${ }^{1} \quad$ XU Gui-Ying ${ }^{1, *} \quad$ TAN Guo-Rong ${ }^{2} \quad$ LÜ Xin ${ }^{2} \quad$ ZHANG Jian ${ }^{2}$ \\ ('Key Laboratory of Colloid and Interface Chemistry, Ministry of Education, Shandong University, Jinan 250100, P. R. China; \\ ${ }^{2}$ State Key Laboratory of Offshore Oil Exploitation, Technology Research Dept. CNOOC Research Center, \\ Beijing 100027, P. R. China)
}

\begin{abstract}
Three poly(propylene oxide)-poly(ethylene oxide) branched block polyethers containing a benzene ring moiety were synthesized, with different molecular weights and propylene oxide/ethylene oxide (PPO/PEO) compositions. Their aggregation behaviors at air/water and oil/water interfaces were investigated by interfacial tension, surface pressure and interfacial dilational rheology methods. Aggregation and the emulsion breaking properties (demulsification) for crude oil were studied based on the polymer PEO content and molecular weight. The demulsification performance of these polyethers and their crosslinked counterparts were compared at different temperatures. The results showed that a polyether with a higher proportion of PEO groups and larger molecular weight occupied a larger area at the air/water interface, reached equilibrium faster, and featured a larger dilational modulus at the oil/water interface. However, the demulsification experiments showed that the polyether with a moderate level of PEO content gave better performance. The cross linking method did not improve demulsification ability in the polyether with large molecular weights. Temperature was also found to have no explicit influence on the demulsification of the cross linked polyethers. This study provides useful data for the selection and application of chemicals used in processing of crude oil.
\end{abstract}

Key Words: Branched block polyether; Interfacial activity; Interfacial dilational rheology; Demulsification

Received: January 29, 2013; Revised: March 25, 2013; Published on Web: March 25, 2013.

"Corresponding author. Email: xuguiying@sdu.edu.cn; Tel: +86-531-88365436; Fax: +86-531-88564750.

The project was supported by the Special Program for Major Research of the Science and Technology, China (2011ZX05024-004-08).

国家科技重大专项基金(2011ZX05024-004-08)资助

C. Editorial office of Acta Physico-Chimica Sinica 


\section{1 引言}

聚氧丙烯/聚氧乙烯(PPO-PEO)类嵌段聚醚大 分子结构有着丰富的可设计性, 如改变 PEO/PPO 比 例、链段长度、支化度以及分子量等, 从而极大地丰 富了此类大分子在体相和界面的自组装行为. ${ }^{1-7}$ PPO-PEO 类嵌段聚醚具有良好的钙㿝分散作用、增 溶作用以及无毒、无臭、无味、无刺激性等特征. 因此 不仅在许多工业领域可以用作消泡剂、乳化剂、破乳 剂、润滑剂、增溶剂、洗涤剂, 而且是化妆品、食品和 医药等产品的重要添加剂. 近年来还发现它们在药 物增溶和缓释、废水处理、介孔材料的制备、动物细 胞培养和生物大分子分离等方面也具有广泛的应用 前景. ${ }^{8-11}$ 所以, 人们对聚醚类高分子表面活性剂的 研究一直很感兴趣..$^{12-15}$ 近年来的研究发现, 非线性 嵌段聚醚的聚集行为不同于线性分子, ${ }^{16-24}$ 而且在微 纳米材料制备、 ${ }^{25}$ 碳纳米管分散稳定 ${ }^{26,27}$ 以及原油破 乳 28,29 等方面已展现出良好的应用前景.

若原油中含有水及溶解于水中的杂质, 则在其 集输和炼制过程中会增加洜、管线和储罐的负荷, 并 可能引起管道系统、泵及生产设备的腐蚀和结垢等 问题; 而排放的水中含油则会造成环境污染和油的 浪费. ${ }^{30-32}$ 尤其是油田进入开发后期, 采出的原油含 水量越来越高, 而强化采油技术的推广应用, 又可能 使采出的原油形成水包油型甚至多层乳状液, 导致 原油的破乳脱水处理更加困难. 因此有关乳化原油 破乳脱水的研究一直是倍受重视的课题.

本文以 2,4,6-三羟甲基苯酚与二乙烯三胺反应 产物为起始剂合成的具有 13 支结构的 PPO-PEO 嵌 段聚醚(简写为 SF) 为研究对象, 通过界面张力、界面 流变性和对原油乳状液的破乳脱水效果的测定, 考 察了其聚集行为和破乳作用对 EO 含量和分子量的 依赖性, 以期为原油集输过程中化学品的选择与应 用提供依据.

\section{2 实验部分}

\section{1 试 剂}

$\mathrm{SF}$ 系列支状嵌段聚醚由本实验室按照与文 献 ${ }^{18-22}$ 相似的方法合成和表征. 其结构如图 1 所示. 采用端基分析法测量了 SF332、SF321 和 SF331 的分 子量, 通过核磁共振氢谱('H NMR) (Bruker AV-400 NMR spectrometer) 分别测得三样品的核磁共振图 (见支撑材料 1), 由此计算得到聚醚分子中 PEO 和 PPO 的含量. 结果表明, PPO 和 PEO 质量分数与设

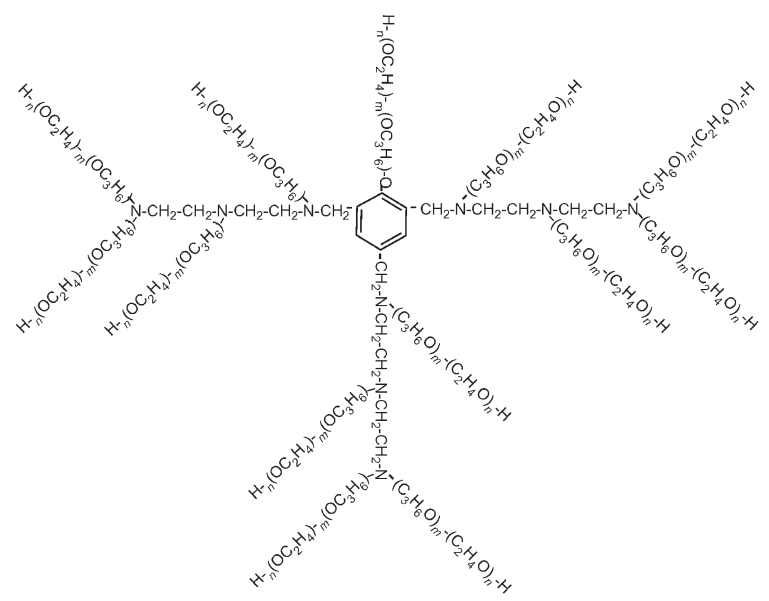

图 $1 \mathrm{SF}$ 系列聚醚(SF332, SF321, SF331)的分子结构示意图

Fig. Molecular structures of the branched block polyethers (SF332, SF321, SF331)

计投料相近. SF 系列聚醚的分子组成相关参数见表 1. SF332、SF321 和 SF331 分子与甲苯二异氧酸酯 (TDI)交联后所得产物分别表示为 SFI332、SFI321 和 SFI331(具体反应见支撑材料 2). 甲苯 $(>99.5 \%$ )、氯 仿( $>99 \%$ )和正庚烷(>98.5\%)等均购于天津标准科技 有限公司. 用于界面性能测试的溶液均用三次蒸馏 水配制.

\section{2 实验方法}

\subsection{1 表面性质测定}

分别称取一定质量的 SF332、SF321 和 SF331 配 制成 1\%(w)的咜备溶液，再分别稀释成不同浓度的 溶液数份, 按文献 ${ }^{18-22}$ 方法在 K12 型表面张力仪(瑞 士KRÜSS 公司)上测定三者的表面张力等温线.

按照文献 ${ }^{33}$ 方法, 在 NIMA 601(英国 NIMA 公 司)矩形双臂压缩微型槽上测定表面压. 亚相为三次 蒸馏水, 铺展液为聚醚的氯仿溶液 (浓度为 $0.05 \mathrm{~g}$. $\mathrm{L}^{-1}$ ). 用微量注射器将一定体积的铺展液均匀滴加 在整个液面上, 滴加时注射器针头与液面的距离应 尽可能小. 进样完成后, 根据进样体积的不同分别挥 发 10-30 $\mathrm{min}$ 后以 $3 \mathrm{~cm}^{2} \cdot \mathrm{min}^{-1}$ 的速率对溶液表面进

表 1 SF 系列支状嵌段聚醚(SF332, SF321, SF331)的分子组 成及相关参数

Table 1 Molecular compositions and parameters of the branched block polyethers (SF332, SF321, SF331)

\begin{tabular}{ccccc}
\hline \multirow{2}{*}{ Polyether } & $\frac{\text { Molecularweight }}{}$ & $w_{\mathrm{PEO}} / \%$ & $w_{\mathrm{PPO}} / \%$ & Cloud point ${ }^{\mathrm{a} /{ }^{\circ} \mathrm{C}}$ \\
\hline SF332 & 24360 & 41 & 49 & 62.5 \\
SF321 & 23140 & 33 & 49 & 34.5 \\
SF331 & 21750 & 22 & 49 & 25.3 \\
\hline
\end{tabular}

${ }^{\mathrm{a}}$ cloud point of $1 \%(w)$ of the polyether aqueous solution 
行压缩, 测得表面压-面积 $(\pi-A)$ 等温线.

表面特性测定均在 $(25.0 \pm 0.1){ }^{\circ} \mathrm{C}$ 下进行.

\section{2 .2 油/水界面性质的测定}

以正庚烷: 甲苯(体积比 7:3)模拟油作为轻相, 不 同浓度聚醚水溶液作为重相. 利用 Tracker 界面流变 仪(法国 Teclis 公司)测定界面张力和扩张流变性. 测 量时将重相放在石英池中, 装有轻相的注射器针头 插入重相溶液中, 通过马达运动来控制注射器形成 一定体积的液滴从而得到油/水界面. 利用 CCD 照 相机采集液滴形状的变化信息,通过软件计算得到 界面张力值; 通过马达控制液滴以一定振幅周期性 扩张收缩即得到相关扩张粘弹性数据. 此测定过程 中所用聚醚浓度均为 $100 \mathrm{mg} \cdot \mathrm{L}^{-1}$.

界面特性测定均在 $(30.0 \pm 0.1){ }^{\circ} \mathrm{C}$ 下进行.

\subsection{3 原油乳状液脱水实验}

称取一定量 SZ36-1 油田的脱水原油于 HT-II 型 混调器(无锡市石油仪器设备厂) 中, 将其置于实验 温度的水浴中恒温 $0.5 \mathrm{~h}$, 按油水体积比为 $7: 4$ 的量 加入纯净水, $1200 \mathrm{r} \cdot \mathrm{min}^{-1}$ 下充分乳化 $5 \mathrm{~min}$. 制得模 拟原油乳状液. 将模拟原油乳状液倒入实验所需的 具塞量筒中分别至 $80 \mathrm{~mL}$. 实验中需同时做平行试 验和空白试验, 于对应的量筒中用移液管加入适量 的样品溶液和参比样溶液. 将加药后的具塞量筒置 于实验温度的恒温水浴中预热 $30 \mathrm{~min}$. 将预热后的 具塞量筒上下手摇 100 次, 左右手量筒交换开塞放 空后再摇 100 次, 然后开塞放空后置于实验温度恒 温水浴中静置 2-3 h. 读取不同时间下脱出水的体 积, 并观察具塞量筒中的油/水界面、中间层状况及 脱出水的颜色. 为了考察聚醚交联是否对其破乳性 能的影响, 分别在 60 和 $70{ }^{\circ} \mathrm{C}$ 时对比研究了 $\mathrm{SF}$ 聚醚

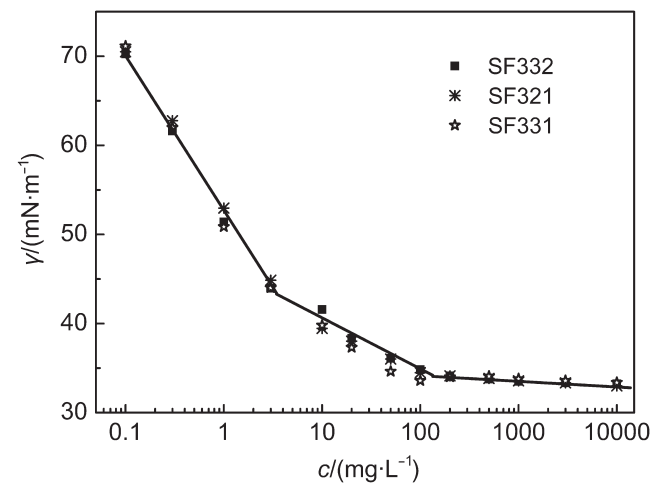

图 $225^{\circ} \mathrm{C}$ 时 $\mathrm{SF}$ 支状聚醚(SF332, SF321, SF331)的表面张 力等温线

Fig.2 Surface tension isotherms of the SF branched polyethers (SF332, SF321, SF331) at $25^{\circ} \mathrm{C}$
交联前后对原油乳状液的脱水效果. 聚醚加量恒定 为 $100 \mathrm{mg} \cdot \mathrm{L}^{-1}$.

\section{3 结果与讨论}

\section{1 表面活性}

大量研究表明, 两亲分子的表面活性可以用水 的表面张力降低的性质来表示. 通常, 两亲分子将 水的表面张力降低 $20 \mathrm{mN} \cdot \mathrm{m}^{-1}$ 时所需的浓度 $\left(c_{20}\right)$ 称 之为降低表面张力的效率, 而将一定浓度下可达到 的最低表面张力称之为降低表面张力的效能. 图 2 示出了SF332、SF321 和 SF331 水溶液的表面张力等 温线, 可以看出, 三种聚醚的 $c_{20}$ 均在 $1 \mathrm{mg} \cdot \mathrm{L}^{-1}$ 左右, 而最低表面张力值 $\gamma_{\mathrm{cmc}}$ 可降至 $33 \mathrm{mN} \cdot \mathrm{m}^{-1}$ 左右, 说明 三者降低水的表面张力的能力和效率相近. 临界胶 束浓度 $(\mathrm{cmc})$ 是表面活性剂的重要特性参数之一, 当 溶液浓度达到 $\mathrm{cmc}$ 值后, 溶液中开始大量形成胶束. 由图 2 可知, 三种聚醚的表面张力等温线均出现双 拐点现象. 研究表明, ${ }^{18-22}$ 双拐点的出现与两亲分子 的分子量分布较宽或者 $\mathrm{cmc}$ 之前形成单分子胶束或 寡聚体有关. 因此, 一般取表面张力等温线上第二 个拐点对应的浓度作为其 $\mathrm{cmc}$. 显然, 三种聚醚的 $\mathrm{cmc}$ 值均为 $100 \mathrm{mg} \cdot \mathrm{L}^{-1}$ 左右. 可能的原因是三种大 分子的结构相同, 疏水链段(PPO)也相同, 只有 PEO 链段有所差异, 而亲水的 PEO 又往往深入水相中, 因此三种聚醚分子在界面上的排布构象相似. ${ }^{34,35}$

为了进一步考察三种聚醚分子在气/液表面上 的聚集与铺展状况, 我们测定了三者的 $\pi-A$ 等温线, 结果如图 3 所示. 显然, 三者均只能形成液态扩张 膜, 说明此类大分子在表面上的排布不是很紧密, 可压缩性较强. 低压时, 聚醚分子以伸展的构象平

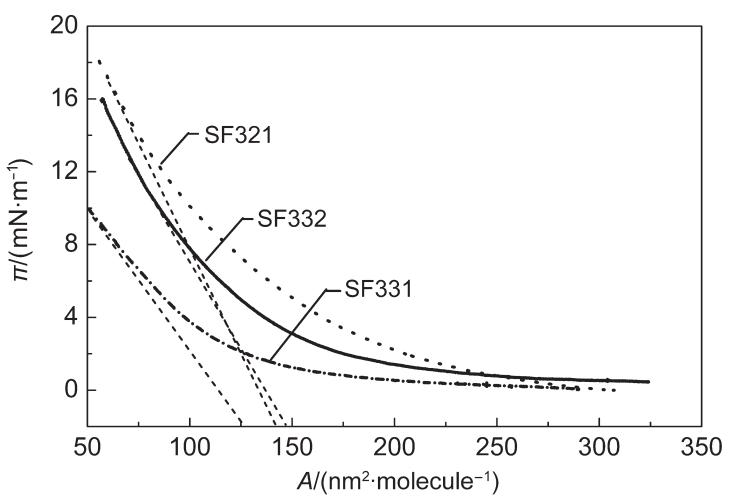

图 $325^{\circ} \mathrm{C}$ 时 SF 支状聚醚(SF332, SF321, SF331)的 $(\pi-A)$ 等温线

Fig.3 Surface pressure-area $(\pi-A)$ isotherms of the SF branched polyethers (SF332, SF321, SF331) at $25^{\circ} \mathrm{C}$ 
躺在表面上, 随着压力增大, 吸附膜被压缩, 亲水的 $\mathrm{PEO}$ 链段逐渐深入到水中, 铺展在表面上的 $\mathrm{PPO}$ 链 段进一步聚集. 但是, 三者的 $\pi-A$ 曲线存在明显差 异, SF332、SF321 和 SF331 所占面积分别是 147、140 和 $126 \mathrm{~nm}^{2} \cdot \mathrm{molecule}^{-1}$, 即 PEO 含量越低, 则其在气/ 液表面上所占据的面积越小. 其原因是: $\mathrm{PPO}$ 链段 含量相同时, 随着聚醚分子 PEO 含量增加, 其在水 中的构象更加伸展, 空间效应阻碍聚醚分子在表面 层的紧密排列, 所以其占据的面积增大. 而 PPO 所 占比例大者, 分子的疏水性强, 易卷曲, 吸附层的可 压缩性强.

\section{2 在油/水界面的聚集行为}

通过动态界面张力测量可以研究聚醚大分子 的吸附动力学. ${ }^{21}$ 图 4 示出了三种聚醚在浓度为 100 $\mathrm{mg} \cdot \mathrm{L}^{-1}$ 时油/水界面张力随时间的变化. 显然, 三者 的界面张力随时间的变化与其分子量大小和 PEO 含量多少的相关性并不明确. 通常, 表面吸附包括 两亲分子由体相到亚表面层的扩散过程、分子从亚 表面层到表面的迁移以及两亲分子在界面上的构 象变化. 分子量愈大, PEO含量愈多, 扩散愈慢, 表 面吸附的时间效应愈长. 但是, 对于油/水界面的吸 附而言, 除了考虑以上因素外, 还应考虑吸附分子 与油相分子的相互作用, 即 PEO 含量少者, 大分子 的疏水性强, 与油分子之间疏水作用强, 即更容易 溶于油相, 从而减弱了其在界面的吸附作用. 而且, PPO 含量比例大者, 大分子更容易发生相互交叠或 卷曲, 则界面上大分子的构象变化复杂, 从而导致 吸附达到平衡的时间较长. 因此, 我们观察到 PEO 含量少者, 其动态界面张力的时间效应反而长. 由 此可见, 大分子在油/水界面的吸附动力学比气/液 表面更为复杂.

界面扩张粘弹性的研究可以提供分子所形成 界面膜的状况, ${ }^{36-38}$ 而研究油/水界面膜的性质有助

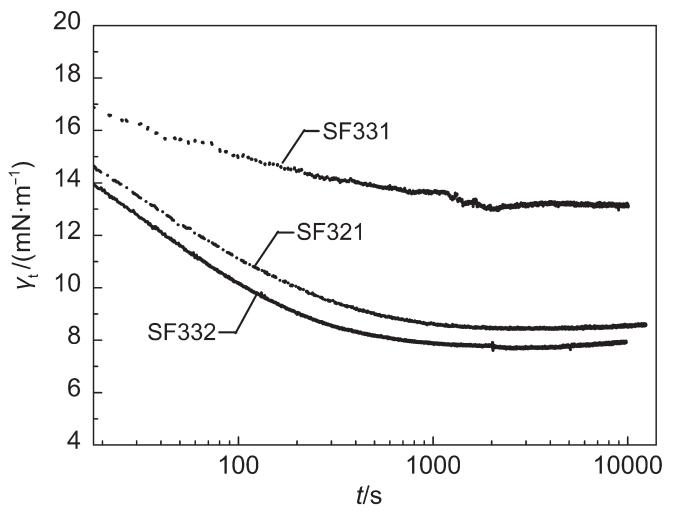

图 $430^{\circ} \mathrm{C}$ 时 SF 支状聚醚(SF332, SF321, SF331)在油/水界 面的动态界面张力曲线

Fig.4 Dynamic interface tension curves of branched polyethers (SF332, SF321, SF331) at oil/water interface at $30{ }^{\circ} \mathrm{C}$

于理解破乳剂的作用机理. ${ }^{39}$ 图 5 示出了三种聚醚浓 度为 $100 \mathrm{mg} \cdot \mathrm{L}^{-1}$ 时在油/水界面的扩张粘弹性与频 率的关系. 由此可以看出, 三者在油/水界面的扩张 模量 $(\varepsilon)$ 和扩张弹性 $\left(\varepsilon_{\mathrm{d}}\right)$ 均随频率 $(\omega)$ 的增大而升高, 但分子量高且 PEO 含量多的 SF332 的 $\varepsilon$ 和 $\varepsilon_{\mathrm{d}}$ 值均高 于 PEO 含量少者, 而且其对 $\omega$ 的依赖性也较大. 这说 明在油/水界面上, 分子量大且 PEO 含量高的聚醚分 子之间相互作用比分子量小者更强, 并且界面受到 扰动时分子构象变化的弛豫过程更加复杂. 尽管 PEO 含量多的聚醚分子在油/水界面的扩张粘性 $\left(\eta \omega_{\mathrm{d}}\right)$ 也较高, 但其值远低于 $\varepsilon_{\mathrm{d}}$ 者, 而且其值随 $\omega$ 增大 变化较小, 甚至呈现出稍微降低的趋势. 这表明三 种聚醚在油/水界面形成的吸附膜均以弹性为主.

\section{3 对原油乳状液的破乳作用}

据报道, 高分子量破乳剂的破乳性能往往好于 低分子量者, 因此, 人们常常将合成的聚醚分子进 行交联, 以提高其分子量. 图 6 示出了 $70^{\circ} \mathrm{C}$ 时支状 聚醚交联前(SF)后(SFI)对油水体积比为 7:4 原油乳 状液的脱水率随时间的变化规律. 由此可见, 油水
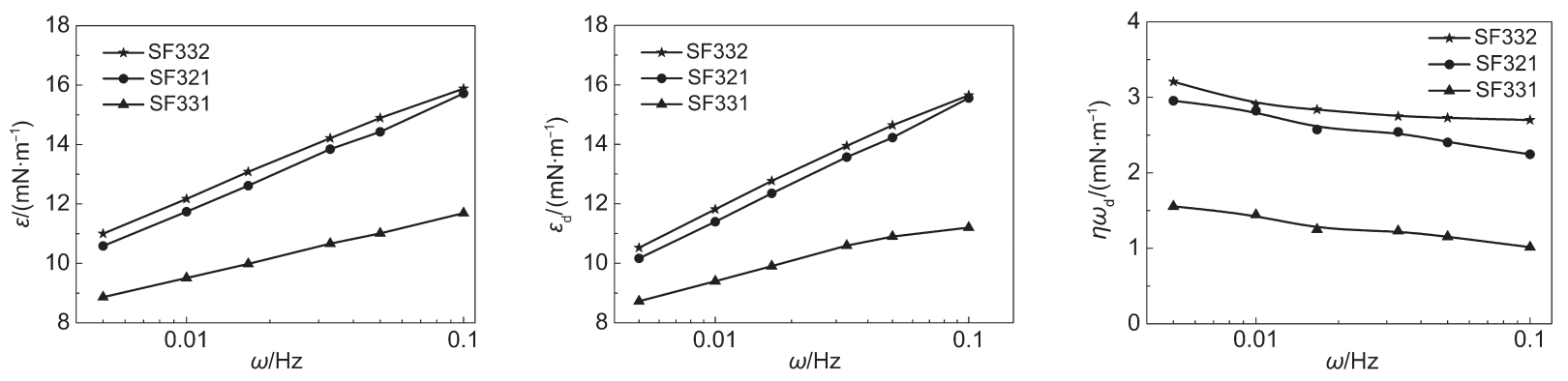

图 $530^{\circ} \mathrm{C}$ 时 $\mathrm{SF}$ 支状聚醚(SF332, SF321, SF331)在油/水界面的流变特性

Fig.5 Dilational rheology of branched polyethers (SF332, SF321, SF331) at oil/water interface at $30{ }^{\circ} \mathrm{C}$

$\varepsilon$ : dilational modulus; $\varepsilon_{\mathrm{d}}$ : dilational flexibility 

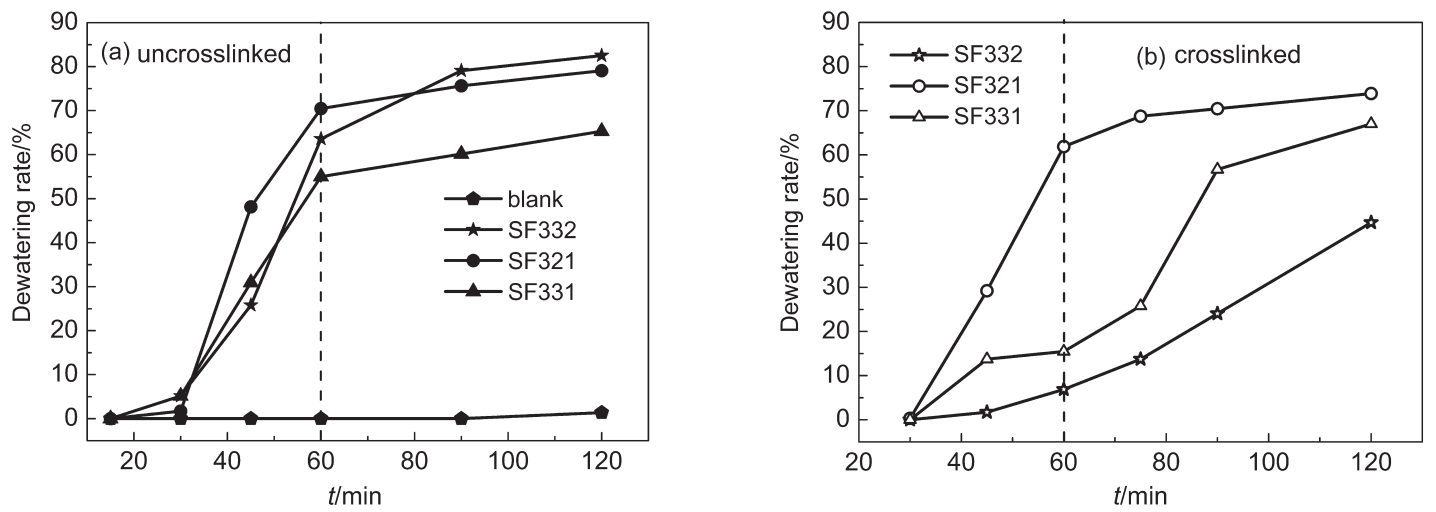

图 $670^{\circ} \mathrm{C}$ 时 $\mathrm{SF}$ 聚醚交联前后对油水体积比为 $7: 4$ 原油乳状液的脱水率随时间的变化

Fig.6 Time dependence of dewatering rate of crude oil emulsion in presence of branched polyethers at oil/water volume ratio of $7: 4$ at $70^{\circ} \mathrm{C}$

体积比为 7:4 原油乳状液的稳定性很好, $120 \mathrm{~min}$ 内 基本无水析出. 加入 $\mathrm{SF}$ 聚醚 $30 \mathrm{~min}$ 后乳状液的脱水 率迅速增大, $60 \mathrm{~min}$ 后乳状液的脱水率基本不再变 化. 但三者可达到的最大脱水率不同, 例如, $60 \mathrm{~min}$ 时 SF321、SF332 和 SF331 的脱水率分别是 $72 \%$ 、 $65 \%$ 和 $57 \%$. 相同条件下, 相应交联产物 SFI332、 SFI321 和 SFI331 的脱水速率明显慢于非交联者, 而 且前者最大脱水率也低于后者, 例如, $60 \mathrm{~min}$ 时 SFI332、SFI321 和 SFI331 的脱水率分别为 $6 \%$ 、62\% 和 $13 \%$. 值得注意的是, SFI321 脱水效果最好, SFI332 的脱水效果最差. 出现此现象的原因可能是 高分子量聚醚的扩散性差, 而破乳剂在油/水界面的 吸附是扩散控制过程, 快速扩散的低分子量破乳剂 可能在破乳中发挥重要作用, 即它们能使乳液脱水 时保持低的动态界面张力. 而且, 交联聚醚大分子 本身在温度较高 $\left(70^{\circ} \mathrm{C}\right)$ 时容易脱水卷缩, 会导致其 对原油乳液中水珠的凝聚能力降低, 因而脱水率和 脱水速率均降低.

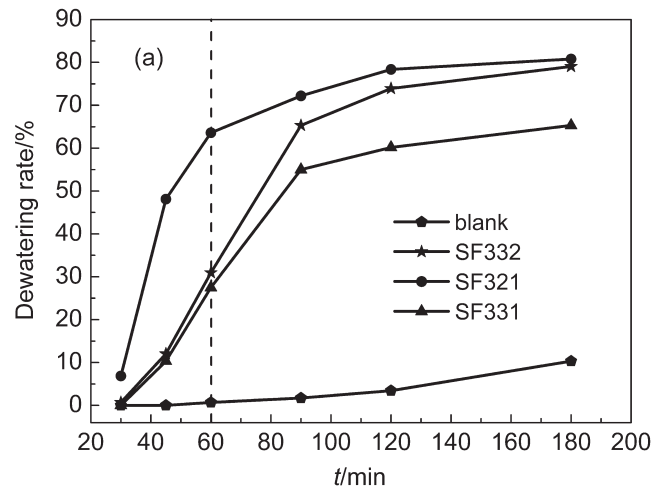

为了探讨温度的影响, 我们考察了 $60^{\circ} \mathrm{C}$ 时 $\mathrm{SF}$ 和 SFI 对油水体积比为 7:4 原油乳状液的脱水率随 时间的变化, 结果如图 7 所示. 显然, $60^{\circ} \mathrm{C}$ 时仍然是 $\mathrm{SF} 321$ 脱水效果最好, SF331 的脱水效果最差, 但 60 $\min$ 时 SF321 的脱水率是 $65 \%, \mathrm{SF} 332$ 和 SF331 的脱 水率仅有 $30 \%$ 左右. 交联产物的破乳脱水速率更 慢, 脱水率更低. 尤其值得注意的是, $60 \mathrm{~min}$ 时 SFI331 的脱水效果好于 SFI332 和 SFI321, 可达 55\%, 而其余两者仅能达 $15 \%$.

乳状液的破乳脱水过程通常为: 破乳剂分子在 油/水界面吸附 $\rightarrow$ 取代 $\rightarrow$ 界面膜破裂 $\rightarrow$ 排液 $\rightarrow$ 水滴 聚并 $\rightarrow$ 油水分离. 因为多支状嵌段聚醚破乳剂分子 极易吸附于油/水界面, 既可顶替原有界面上的沐青 质、胶质等活性分子, 又可同时 “抓住” 原油中的几 个水滴, 导致多个水滴聚并, 则油水能够产生快速 相分离, 从而观察到其快速的脱水破乳现象. ${ }^{28,29}$ 温 度改变可从两个方面影响聚醚分子对原油乳状液 的破乳效果: 一是温度升高时, 导致体系中分子的

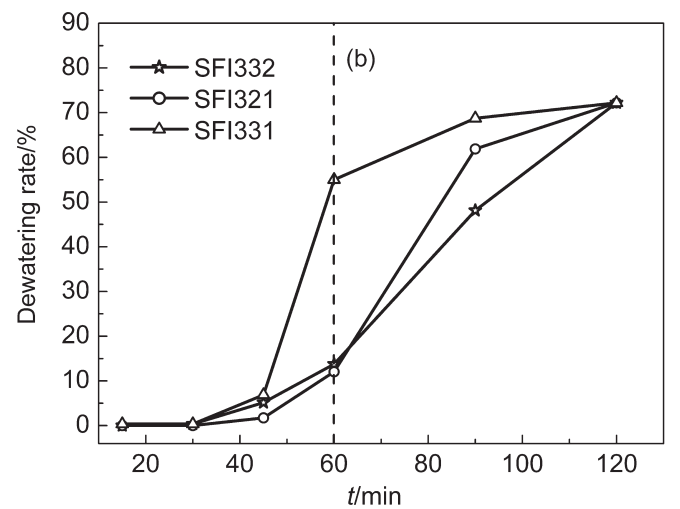

图 $760^{\circ} \mathrm{C}$ 时 $\mathrm{SF}$ 交联前后聚醚对油水体积比为 7:4 原油乳状液的脱水率随时间的变化

Fig.7 Time dependence of dewatering rate of crude oil emulsion in presence of branched polyethers at oil/water volume ratio of $7: 4$ at $60^{\circ} \mathrm{C}$

(a) branched polyethers (SF); (b) crosslinked branched polyethers (SFI) 
热运动加快, 有利于大分子向油/水界面的扩散以及 水滴之间的碰撞聚集, 因而有助于乳状液破乳脱 水; 二是聚醚分子属于非离子型两亲分子, 温度升 高会导致大分子与水分子之间的氢键破坏, 则大分 子链自身会脱水卷曲, 因而不利于其对水滴的凝 聚. 对于 $70^{\circ} \mathrm{C}$ 时 $\mathrm{SF}$ 对原油乳状液的脱水效果好于 $60{ }^{\circ} \mathrm{C}$ 者, 可能归因于温度高时热运动导致体系中大 分子和水滴快速碰撞. 由于聚醚与交联剂均为多官 能团化合物, 两者反应极易形成网状甚至体型结构 的分子, 导致其水溶性变差(SFI 为非水溶性两亲分 子, 应用时溶解于甲醇中), 因而观察到 SFI 表现出 相反的结果, 即交联后其分子量增大, 在水中分散, 扩散能力降低, 而且温度高时其分子的疏水性增 强, 导致其对水滴的凝聚能力降低. 此现象与文献 ${ }^{40}$ 结果相似. 至于 $60^{\circ} \mathrm{C}$ 时 SFI331 对原油乳状液的脱 水效果明显好于 $70{ }^{\circ} \mathrm{C}$ 者, 很可能与分子量和 PEO 基团相对较低的 SF331 与交联剂反应所形成的交联 产物分子量也相对较低有关.

从 $\mathrm{SF}$ 系列聚醚的界面聚集行为及其对原油乳 状液的破乳脱水效果来看, 不同 PEO 含量的聚醚 降低界面张力及油/水界面膜扩张粘弹性的能力与 其破乳效果之间不存在明确的对应关系, 这也与文 献 ${ }^{39}$ 报道的结果相类似. 原因可能是 $\mathrm{SF}$ 聚醚的聚集 行为研究部分采用的模型油成分简单而实际原油 成分较为复杂. 对于原油乳状液体系, 天然乳化剂 分子会参与油/水界面膜的形成, 因此, 油/水界面膜 的性质与成分简单的模型油体系不能较好地吻合.

\section{4 结 论}

所合成的 SF 系列支状聚醚具有良好的表面活 性, 但由于三种聚醚具有相同的分子结构和相同的 PPO 含量, 其表面活性没有明显差异. 三种聚醚在 气/液界面上的排布不是很紧密, 形成的界面膜有较 强的可压缩性, 其中 PEO 含量高者在水中的构象更 加伸展, 其分子在界面上的极限占据面积也较大. 动态油/水界面张力与 $\mathrm{SF}$ 系列聚醚分子量大小和 PEO 含量多少的相关性并不明确, 因为除了扩散作 用还应考虑界面吸附分子与油相分子的相互作用, PEO 含量小者疏水性强, 与油分子之间疏水作用 强, 并且分子更容易发生相互交叠或卷曲, 在界面 上的构象变化复杂, 从而导致吸附达到平衡的时间 较长. SF 系列聚醚分子在油/水界面形成的吸附膜 以扩张弹性为主, 分子量大且 PEO含量高者界面扩
张模量和扩张粘弹性均高于 PEO 含量低者. 这说明 在油/水界面, 前者的分子之间相互作用比后者更强 并且界面受到扰动时分子构象变化的弛豫过程更 加复杂. 原油乳状液的破乳脱水实验表明, EO 含量 为 $33 \%$ 的 SF 聚醚破乳效果优于其它二者, 相同条件 下, $\mathrm{SF}$ 聚醚交联产物的脱水速率慢于非交联者, 而 且其最大脱水率也低于非交联者, 说明多支化聚醚 分子能够满足某些原油乳状液的破乳要求, 不需通 过交联提高其分子量.

\section{References}

(1) Zana, R.; Marques, C.; Johner, A. Adv. Colloid Interface Sci. 2006, 123-126, 345.

(2) Arleth, L.; Svensson, B.; Mortensen, K.; Pedersen, J. S.; Olsson, U. Langmuir 2007, 23, 2117. doi: 10.1021/1a0625704

(3) Ganguly, R.; Kumbhakar, M.; Aswal, V. K. J. Phys. Chem. B 2009, 113, 9441. doi: 10.1021/jp900535f

(4) Perry, C. C.; Sabir, T. S.; Livingston, W. J.; Milligan, J. R.; Chen, Q.; Maskiewicz, V.; Boskovic, D. S. J. Colloid Interface Sci. 2011, 354, 662. doi: 10.1016/j.jcis.2010.10.028

(5) Liang, X. F.; Guo, C.; Ma, J. H.; Wang, J.; Chen, S.; Liu, H. Z. J. Phys. Chem. B 2007, 111, 13217. doi: 10.1021/jp074990n

(6) Zhang, X. Q.; Yuan, S. L.; Wu, J. Macromolecules 2006, 39, 6631. doi: 10.1021/ma061201b

(7) Cao, X. R.; Xu, G. Y.; Li, Y. M.; Zhang, Z. Q. J. Phys. Chem. A 2005, 109, 10418. doi: 10.1021/jp053636r

(8) Chen, S.; Guo, C.; Hu, G. H.; Wang, J.; Ma, J. H.; Liang, X. F.; Zheng, L.; Liu, H. Z. Langmuir 2006, 22, 9704. doi: 10.1021/ la061093m

(9) Wang, F.; Xu, G. Y.; Zhang, Z. Q.; Xin, X. Colloids Surf. A 2005, 259, 151. doi: 10.1016/j.colsurfa.2005.02.025

(10) Wang, L.; Chen, X.; Zhan, J.; Chai, Y.; Yang, C.; Xu, L.; Zhuang, W.; Jing, B. J. Phys Chem. B 2005, 109, 3189. doi: $10.1021 / j p 0449152$

(11) Huang, Y.; Cai, H. Q.; Yu, T.; Sun, X. L.; Tu, B.; Zhao, D. Y. Chem. Asian J. 2007, 2, 1282.

(12) Chiappetta, D. A.; Alvarez-Lorenzo, C.; Rey-Rico, A.; Taboada, P.; Concheiro, A.; Sosnik, A. Eur. J. Pharm. Biopharm. 2010, 76, 24. doi: 10.1016/j.ejpb.2010.05.007

(13) Su, Y. L.; Wang, J.; Liu, H. Z. Macromolecules 2002, 35, 6426. doi: $10.1021 / \mathrm{ma} 0105284$

(14) Bae, K. H.; Lee, Y.; Park, T. G. Biomacromolecules 2007, 8, 650. doi: 10.1021/bm0608939

(15) Tsui, H. W.; Wang, J. H.; Hsu, Y. H.; Chen, L. J. Colloid Polym Sci. 2010, 288, 1687. doi: 10.1007/s00396-010-2308-5

(16) Kadam, Y.; Singh, K.; Marangoni, D. G.; Ma, J. H.; Aswal, V. K.; Bahadur, P. Colloids Surf. A 2010, 369, 121. doi: 10.1016/ j.colsurfa.2010.08.010

(17) Kadam, Y.; Singh, K.; Marangoni, D. G.; Ma, J. H.; Aswal, V. 
K.; Bahadur, P. J. Colloid Interface Sci. 2010, 351, 449. doi: 10.1016/j.jcis.2010.07.046

(18) Xin, X.; Xu, G. Y.; Zhang, Z. Q.; Chen, Y. J.; Wang, F. Eur. Polym. J. 2007, 43, 3106. doi: 10.1016/j.eurpolymj.2007.04.005

(19) Liu, T.; Xu, G. Y.; Gong, H. J.; Pang, J. Y.; He, F. Langmuir 2011, 27, 9253. doi: 10.1021/la201676u

(20) Gong, H. J.; Xu, G. Y.; Shi, X. F.; Liu, T.; Sun, Z. W. Colloid Polym. Sci. 2010, 288, 1581. doi: 10.1007/s00396-010-2294-7

(21) Gong, H. J.; Xu, G. Y.; Ding, H.; Shi, X. F.; Tan, Y. B. Eur. Polym. J. 2009, 45, 2540. doi: 10.1016/j.eurpolymj.2009.05.027

(22) Gong, H. J.; Xu, G. Y.; Liu, T.; Xu, L.; Zhai, X. R. Langmuir 2012, 28, 13590. doi: 10.1021/la303430c

(23) Cao, X. R.; Xu, G. Y.; Yuan, S. L.; Gao, B. Y. Soft Matter 2011, 7, 9035. doi: 10.1039/c1sm05319a

(24) De Lisi, R.; Giammona, G.; Lazzara, G. Milioto, S. J. Colloid Interface Sci. 2011, 354, 749. doi: 10.1016/j.jcis.2010.11.075

(25) Wang, F.; Xu, G. Y.; Zhang, Z. Q.; Xin, X. Eur. J. Inorg. Chem. 2006, $1,109$.

(26) Xin, X.; Xu, G. Y.; Zhao, T. T.; Zhu, Y. Y.; Shi, X. F.; Gong, H. J.; Zhang, Z. Q. J. Phys. Chem. C 2008, 112, 16377. doi: 10.1021/jp8059344

(27) Gong, H. J.; Xu, G. Y.; Liu, T.; Pang, J. Y.; Dou, W. L.; Xin, X. Colloid Polym. Sci. 2011, 289, 933. doi: 10.1007/s00396011-2419-7

(28) Zhang, Z. Q.; Xu, G.Y.; Wang, F.; Dong, S. L.; Li, Y. M. J. Colloid Interface Sci. 2004, 277, 464. doi: 10.1016/j.jcis.2 004.04 .035

(29) Zhang, Z. Q.; Xu, G. Y.; Wang, F.; Dong, S. L.; Chen, Y. J.
J. Colloid Interface Sci. 2005, 282, 1. doi: 10.1016/j.jcis. 2004.08.144

(30) Li, M. Y. Acta Petrolei Sinica (Petroleum Processing Section) 1995, 11, 1. [李明远. 石油学报 (石油加工), 1995, 11, 1.]

(31) Wu, X. Energy Fuels 2003, 17, 179. doi: 10.1021/ef020098y

(32) Xia, L. X.; Lu, S. W.; Cao, G. Y. J. Colloid Interface Sci. 2004, 271, 504. doi: 10.1016/j.jcis.2003.11.027

(33) Liu, H. G.; Qian, D. J.; Feng, X. S.; Xue, Q. B.; Yang, K. Z. Langmuir 2000, 16, 5079. doi: 10.1021/1a991253c

(34) Alexandridis, P.; Athanassiou, V.; Fukuda, S.; Hatton, T. A. Langmuir 1994, 10, 2604. doi: 10.1021/la00020a019

(35) De Lisi, R.; Milioto, S. Langmuir 2000, 16, 5579. doi: 10.1021/ la991586+

(36) Noskov, B. A.; Lin, S. Y.; Loglio, G.; Rubio, R. G.; Miller, R. Langmuir 2006, 22, 2647. doi: 10.1021/1a052662d

(37) Wang, Y. Y.; Zhang, L.; Sun, T. L.; Zhao, S.; Yu, J. Y. J. Colloid Interface Sci. 2004, 270, 163. doi: 10.1016/j.jcis.2003.09.046

(38) Wang, Y. Y.; Dai, Y. H.; Zhang, L.; Tang, K.; Luo, L.; Gong, Q. T.; Zhao, S.; Li, M. Z.; Wang, E. J.; Yu, J. Y. J. Colloid Interface Sci. 2004, 280, 76. doi: 10.1016/j.jcis.2004.07.016

(39) Kang, W. L.; Zhang, H. Y.; Li, D. S.; Wu, Z. L.; Li, M. Y.; Gao, H. M. Acta Phys. -Chim. Sin. 2004, 20, 194. [康万利, 张红艳, 李道山, 吴肇亮, 李明远, 高慧梅. 物理化学学报, 2004, 20, 194.] doi: 10.3866/PKU.WHXB20040218

(40) Gong, H. J.; Hu, G. Y.; Chen, G. X. Spec. Petrochem. 2000, No.4, 1. [龚惠娟, 胡耿源, 陈关喜. 精细石油化工, 2000, No.4, 1.] 
Supplementary Information for Acta Phys. -Chim. Sin. 2013, 29 (6): 1253-1259 doi: 10.3866/PKU.WHXB201303251

\title{
支状嵌段聚醚的界面聚集行为及对原油乳状液的破乳作用
}

\author{
翟雪如 $^{1} \quad$ 刘腾 ${ }^{1}$ 徐桂英 ${ }^{1, *}$ 檀国荣 ${ }^{2} \quad$ 吕釒金 $^{2}$ 张健 $^{2}$ \\ $\left({ }^{1}\right.$ 山东大学胶体与界面化学教育部重点实验室, 济南 250100； ${ }^{2}$ 中海油研究总院技术研发中心, \\ 海洋石油高效开发国家重点实验室, 北京 100027)
}

\section{Aggregation Behavior of Branched Block Polyethers at Interface and Their Demulsification for Crude Oil Emulsion}

\author{
ZHAI Xue-Ru $^{1} \quad$ LIU Teng $^{1} \quad$ XU Gui-Ying $^{1, *} \quad$ TAN Guo-Rong $^{2} \quad$ Lü Xin ${ }^{2}$ \\ ZHANG Jian $^{2}$
}

( ${ }^{1}$ Key Laboratory of Colloid and Interface Chemistry, Ministry of Education, Shandong University, Jinan 250100,

P. R. China; ${ }^{2}$ State Key Laboratory of Offshore Oil Exploitation, Technology Research Dept. CNOOC Research Center, Beijing 100027, P. R. China)

*Corresponding author. Email: xuguiying@sdu.edu.cn; Tel: +86-531-88365436; Fax: +86-531-88564750. 
1. $\mathrm{SF}$ 聚梄核磁谱图

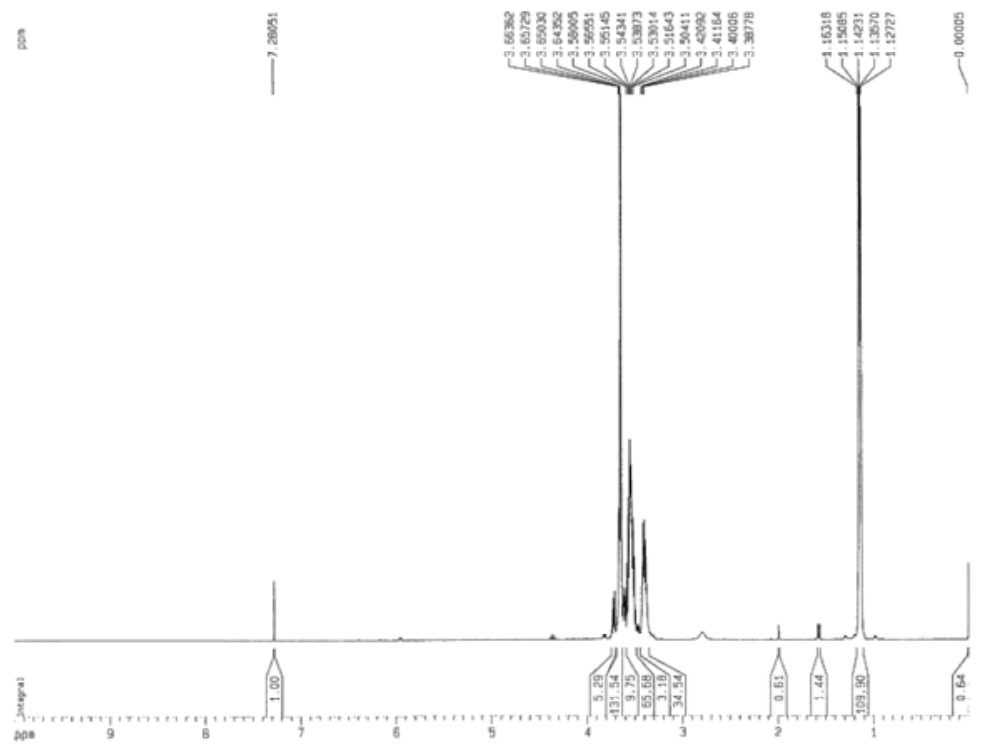

a. SF332 核磁共振图

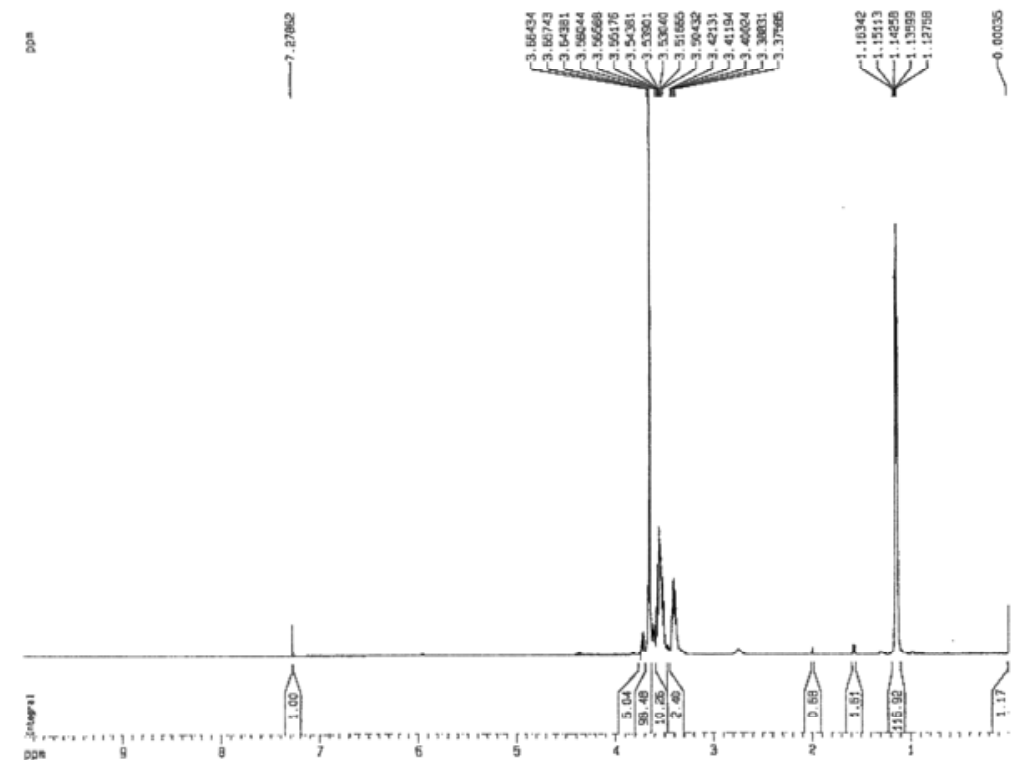

b. SF321 核磁共振图 


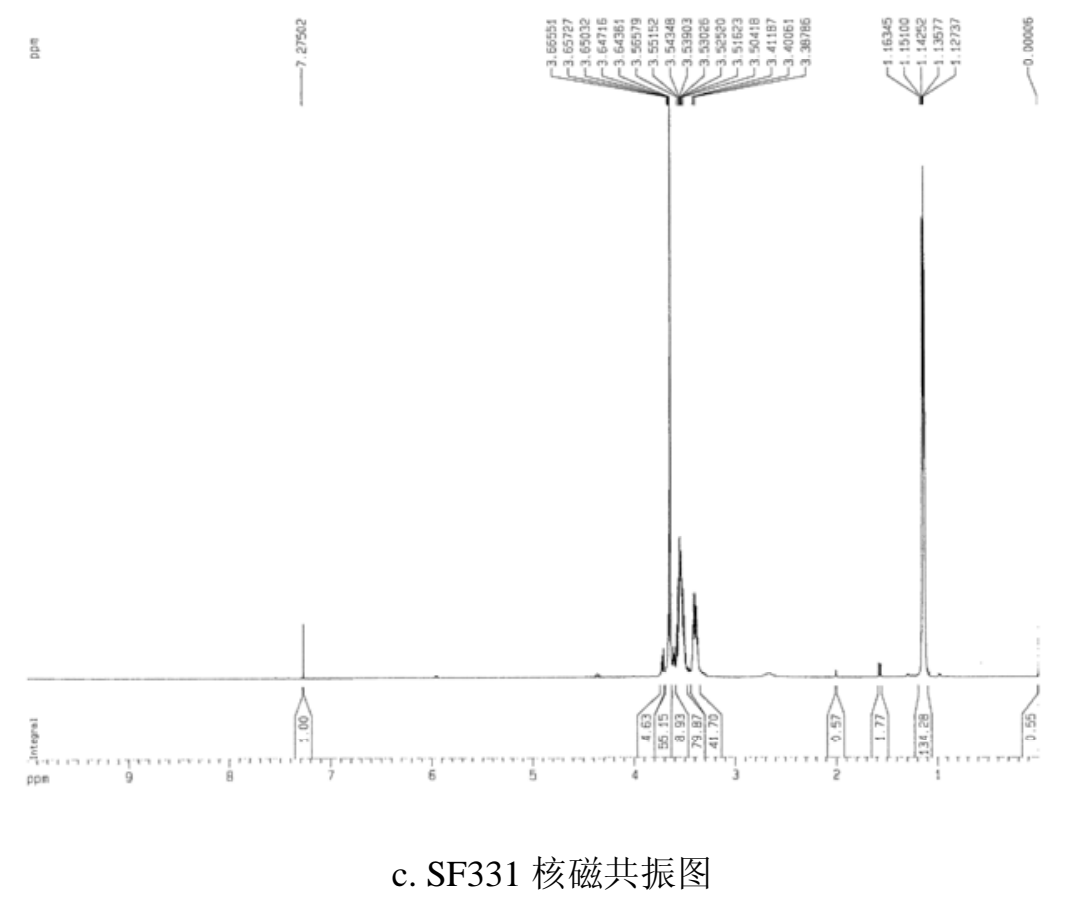

2. SF 聚醚的交联反应

称取一定量的 SF 样品, 经 $\mathrm{CaH}_{2}$ 回流脱水后的甲苯 $80 \mathrm{~mL}$, 置于 $250 \mathrm{~mL}$ 三 颈瓶中, 升温至 $50{ }^{\circ} \mathrm{C}$ 时, 边搅拌边缓慢滴加一定量的 TDI（溶于脱水后的甲苯 中），约 $1 \mathrm{~h}$ 滴完，滴加完毕后，继续摚拌半小时，整个过程控制温度为 $50 \sim 60$ ${ }^{\circ} \mathrm{C}$ 。SF 系列嵌段聚醚水溶性良好, 其与 TDI 的交联产物为油溶性破乳剂, 以 SFI 表示之。 